\title{
Exogenous marker-engineered mesenchymal stem cells detect cancer and metastases in a simple blood assay
}

\author{
Linan Liu'1,2,3,4,5, Shirley X. Zhang ${ }^{1,2,3,4,5}$, Rangoli Aeran 1,2,3,4,5, Wenbin Liao ${ }^{1,2,3,4,5}$, Mengrou Lu ${ }^{1,2,3,4,5}$, \\ George Polovin ${ }^{1,2,3,4,5,6}$, Egest J. Pone ${ }^{1,2,3,4,5}$ and Weian Zhao ${ }^{1,2,3,4,5^{*}}$
}

\begin{abstract}
Introduction: Mesenchymal stem cells (MSCs) are adult multipotent stem cells that possess regenerative and immunomodulatory properties. They have been widely investigated as therapeutic agents for a variety of disease conditions, including tissue repair, inflammation, autoimmunity, and organ transplantation. Importantly, systemically infused MSCs selectively home to primary and metastatic tumors, though the molecular mechanisms of tumor tropism of MSCs remain incompletely understood. We have exploited the active and selective MSCs homing to cancer microenvironments to develop a rapid and selective blood test for the presence of cancer.
\end{abstract}

Methods: We tested the concept of using transplanted MSCs as the basis for a simple cancer blood test. MSCs were engineered to express humanized Gaussia luciferase (hGluc). In a minimally invasive fashion, hGluc secreted by MSCs into circulation as a reporter for cancer presence, was assayed to probe whether MSCs co-localize with and persist in cancerous tissue.

Results: In vitro, hGluc secreted by engineered MSCs was detected stably over a period of days in the presence of serum. In vivo imaging showed that MSCs homed to breast cancer lung metastases and persisted longer in tumor-bearing mice than in tumor-free mice $(P<0.05)$. hGluc activity in blood of tumor-bearing mice was significantly higher than in their tumor-free counterparts $(P<0.05)$.

Conclusions: Both in vitro and in vivo data show that MSCs expressing hGluc can identify and report small tumors or metastases in a simple blood test format. Our novel and simple stem cell-based blood test can potentially be used to screen, detect, and monitor cancer and metastasis at early stages and during treatment.

\section{Introduction}

Cancer is a leading cause of human morbidity and mortality, and its origins, biomarkers, and detection remain difficult to pinpoint [1]. Although early detection has proven to be a useful and often necessary first step to effectively manage and treat cancer [2], it remains a challenge at early stages to identify cancer, especially small tumors and metastases which account for over $90 \%$ of cancer mortality [3, 4]. Methods of cancer detection based on imaging are non-invasive, but common

\footnotetext{
* Correspondence: weianz@uci.edu

'Department of Pharmaceutical Sciences, University of California, Irvine, 845 Health Sciences Road, Irvine, CA 92697, USA

${ }^{2}$ Department of Biomedical Engineering, University of California, Irvine, 845 Health Sciences Road, Irvine, CA 92697, USA

Full list of author information is available at the end of the article
}

drawbacks include high cost, low specificity or resolution, and the use of potentially irritating contrast agents [2]. For instance, positron emission tomography (PET), computed tomography $(\mathrm{CT})$, and their combinations (PET-CT) are widely used for identifying and staging tumors but require high doses of ionizing radiation and have limited specificity and resolution [5]. Other imaging modalities, such as magnetic resonance imaging (MRI) and ultrasound, do not use radiation but are still unable to achieve spatial resolution smaller than several millimeters $[6,7]$. On the other hand, tissue biopsies are invasive and suffer from false negatives for heterogeneous tumors, and obtaining biopsies from multiple small disseminated tumors (e.g., metastases) is impractical. Cancer screening also uses tests for biomarkers, including circulating tumor cells, exosomes, proteins, and nucleic acids. Recently, scientists 


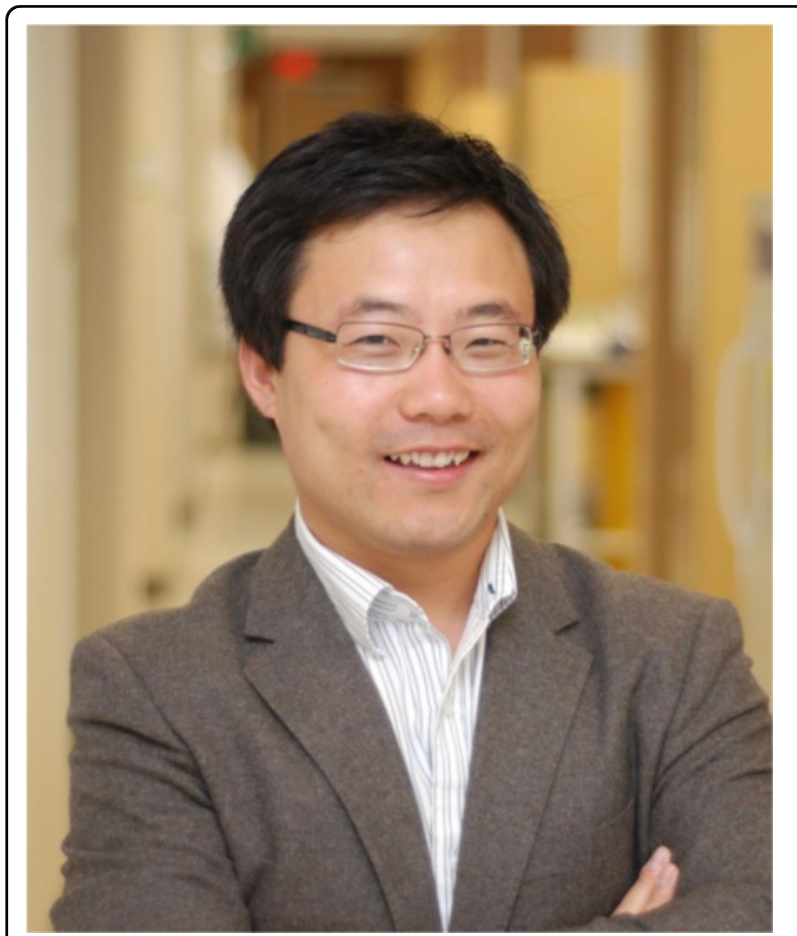

Box 1. About Weian Zhao

Weian Zhao is an assistant professor at the Department of

Pharmaceutical Sciences, University of California, Irvine. He completed

his B.Sc. and M.Sc. in chemistry at Shandong University, where he

studied polymer, surface, and colloidal chemistry. In 2008, he received

his Ph.D. in chemistry at McMaster University, where he focused on

the use of functional nucleic acid to structure gold nanoparticles to

construct well-defined nanostructures and biosensors. He then

completed a Human Frontier Science Program Postdoctoral Fellow

at Harvard Medical School, Brigham and Women's Hospital and MIT,

where he learned stem cell trafficking, and cell engineering for

diagnostics and therapeutics. His current research focuses on the

development of novel molecular, nano-, and micro-engineered tools

for stem cell therapy and regenerative medicine, diagnosis and

in vivo imaging, and elucidating stem cell and cancer biology.

have developed nanoparticle-based synthetic biomarkers composed of mass-encoded peptides that can be released upon tumor protease cleavage and then detected in urine $[8,9]$. Such approaches, however, still rely on passive delivery of nanoparticles to tumors via the enhanced permeability and retention (EPR) effect and on limited types of endogenous proteins, both of which are cancer typespecific. More recently, scientists have also reported a probiotic microbe-based system to deliver synthetic biomarker for cancer detection in urine [10]. Nevertheless, cancer biomarker discovery has led to only a few biomarkers used in clinical diagnosis since cancer biomarkers frequently suffer from low sensitivity and specificity [11].
In particular, cancer heterogeneity and evolution make it challenging to rely on molecular biomarkers for cancer detection [1]. For example, the commonly used cancer biomarkers prostate-specific antigen for prostate cancer and $B R C A 1 / 2$ gene mutations for breast cancer can identify only about $25 \%$ and $10 \%$ to $25 \%$ of the patients in each cancer type, respectively [12]. Indeed, it has been widely accepted that a single biomarker typically lacks the sensitivity and specificity that are necessary for useful diagnosis. Intriguingly, recent research indicates that most cancers are caused by stochastic events rather than predictable mutations [13]. Thus, finding biomarkers that recognize multiple types of cancers with no common genetic basis is likely less promising than previously thought. In summary, there is clearly an unmet clinical need for sensitive early-stage cancer and metastasis tests that can "universally" identify many types of cancers independently of specific biomarkers from healthy controls and other conditions that share similar symptoms (e.g., inflammation) as well as to discriminate different (sub)types of cancers at different stages.

Cells, including immune and stem cells, act as autonomous and adaptive agents and these properties have recently been used for cancer treatment and drug delivery [14-17]. In particular, mesenchymal stem (or stromal) cells (MSCs) have been tested as therapeutic agents because of their intrinsic regenerative and immunomodulatory features [18-23]. MSCs are under investigation for treating a wide array of diseases, including diabetes, myocardial infarction, stroke, and autoimmune diseases [24-26]. MSCs are also the world's first manufactured stem cell product to receive clinical approval (i.e., Prochymal manufactured by Osiris (Columbia, MD, USA) was approved in Canada to treat graft-versus-host disease) [26], suggesting that they may be a safe source for diagnostic and therapeutic uses in humans. Importantly, systemically infused MSCs preferentially home to and integrate with tumors, including both primary tumors and metastases in different anatomical locations [24]. As we have recently reviewed [22], mounting evidence now suggests that MSCs possess leukocyte-like, active homing mechanisms for tumor tropism involving a variety of adhesion molecules (e.g., P-selectin and vascular cell adhesion molecule-1) and tumor-derived cytokines, chemokines, and growth factors (e.g., CXCL12 and platelet-derived growth factor). This selective and active homing ability makes MSCs appealing vectors for localized delivery of therapeutics to treat cancers, including gliomas, melanomas, breast cancer, and lung metastases, in ongoing clinical trials $[15,24]$. In addition, MSCs engineered with probes (such as luciferase) have been used to detect and image tumors in situ [19, 27]. However, imaging methods such as PET/single-photon emission computed tomography 


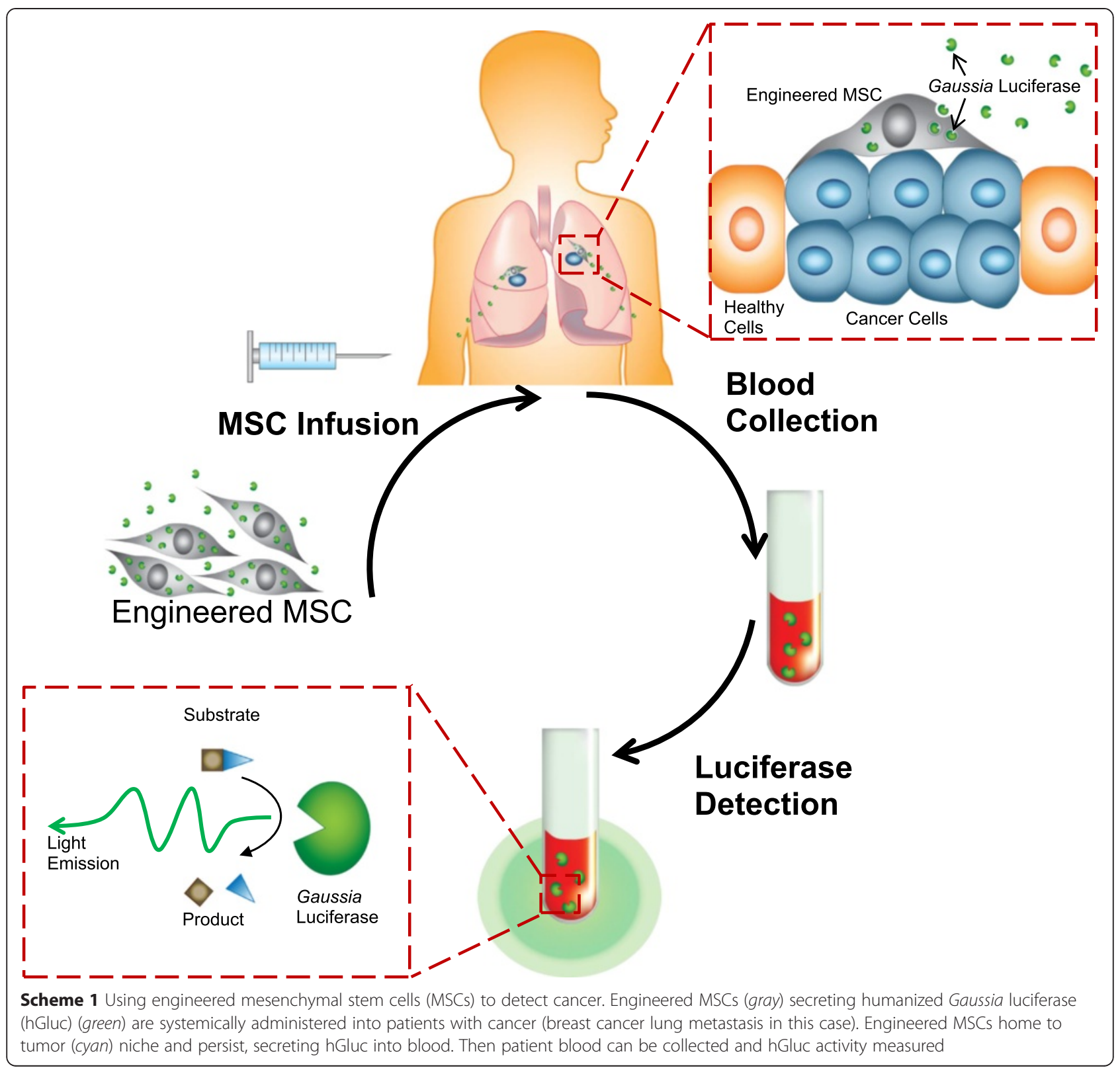

and MRI, which are currently used for cell tracking after infusion are limited by the same aforementioned disadvantages of cancer detection [2].

In this article, we present the concept of using exogenous MSCs as the basis for a simple cancer blood test (Scheme 1). Here, we hypothesize that, owing to their tumor tropism property, MSCs engineered with a secreted reporter can actively and specifically home to tumor sites regardless of the type and location of the tumors and persist there longer compared with MSCs in healthy microenvironments. MSCs engineered to express humanized Gaussia luciferase (hGluc) [28-31] were systemically administered to mice harboring breast cancer cells, exhibited tumor tropism and persistence, and secreted hGluc into the bloodstream of tumor-bearing mice. Thus, MSCs engineered with secreted reporters can potentially be developed into a blood test for broad cancer screening and monitoring.

\section{Methods}

\section{Cell lines and cell culture}

Human bone marrow MSCs were obtained from the Texas A\&M Health Science Center and were expanded to within passages 3-6. The cells were routinely maintained in minimum essential medium alpha (MEM $\alpha$ ) (Life Technologies, Carlsbad, CA, USA) supplemented with $15 \%$ fetal bovine serum (FBS) (Atlanta Biologicals, Norcross, GA, USA) and $1 \%$ penicillin-streptomycin 
(PenStrep) (100 U/ml; Life Technologies) at $37{ }^{\circ} \mathrm{C}$ in a humidified incubator containing $5 \% \mathrm{CO}_{2}$. The human breast cancer cell line MDA-MB-231 was obtained from American Type Culture Collection (ATCC) (Manassas, VA, USA). These cells were grown in Leibovitz's L-15 medium containing L-glutamine (Corning, Corning, NY, USA) and supplemented with $10 \% \mathrm{FBS}$ and $1 \mathrm{U} / \mathrm{ml}$ PenStrep at $37{ }^{\circ} \mathrm{C}$ in a humidified incubator without $\mathrm{CO}_{2}$. The human colon cancer cell line LoVo was obtained from ATCC. These cells were grown in Kaighn's Modification of Ham's F-12 Medium (F-12 K; ATCC) and supplemented with $10 \% \mathrm{FBS}$ and $1 \mathrm{U} / \mathrm{ml}$ PenStrep at $37{ }^{\circ} \mathrm{C}$ in a humidified incubator with $5 \% \mathrm{CO}_{2}$. The 293 T-LV cell line (GenTarget, San Diego, CA, USA) was cultured in Dulbecco's modified Eagle's medium (DMEM) (Life Technologies) supplemented with $15 \%$ FBS, nonessential amino acid (NEAA) (1X, $100 \mathrm{U} / \mathrm{ml}$; Life Technologies), and $1 \mathrm{U} / \mathrm{ml}$ PenStrep at $37^{\circ} \mathrm{C}$ in a humidified incubator containing $5 \% \mathrm{CO}_{2}$. All the cell experiments and procedures were performed after the approval from the University of California, Irvine (UCI) Institutional Biosafety Committee (protocol number 2012-1412).

\section{Generation of lentiviral vectors}

The following lentiviral (LV) vectors were used in this study: LV-eGFP, LV-Fluc-tdT, and LV-hGluc. The sequences of interest from pUCBB-eGFP (\#32548; Addgene, Cambridge, MA, USA), pcDNA3.1(+)/Luc2=tdT (\#32904; Addgene), and pSV40-Gluc (New England BioLabs, Ipswich, MA, USA) were cloned into the promoterless LV transfer vector LV-PL4 (GenTarget).

\section{Lentiviral transduction}

All LV constructs were packaged (pMD2.G, \#12259; pRSVRev, \#12253; pMDLg/pRRE, \#12251; all from Addgene) as LV vectors in 293 T-LV cells [32] by using Lipofectamine LTX and PLUS ${ }^{\text {mix }}$ Reagents (Life Technologies). MSCs and breast cancer cells were transduced with LVs by incubating virions in a culture medium containing $100 \mu \mathrm{g} / \mathrm{ml}$ protamine sulfate (Sigma-Aldrich, St. Louis, MO, USA). After selection with medium containing $10 \mu \mathrm{g} / \mathrm{ml}$ Puromycin (MP Biomedicals, Santa Ana, CA, USA), cells were visualized for fluorescent protein expression by using fluorescence microscopy.

\section{In vitro bioluminescence assays}

LV-Fluc-tdT MSCs (Fluc-tdT-MSCs) expressing firefly luciferase (Fluc) or LV-hGluc MSCs (hGluc-MSCs) expressing humanized Gaussia luciferase (hGluc) were seeded in serially diluted concentrations. After the cells were washed with PBS (Lonza, Basel, Switzerland), luciferase substrates $(150 \mu \mathrm{g} / \mathrm{ml}$ D-luciferin for Fluc, PerkinElmer, Waltham, MA, USA, or $20 \mu \mathrm{M}$ coelenterazine (CTZ) for hGluc, NanoLight Technologies, Pinetop, AZ, USA) were added and the activities of Fluc and hGluc were imaged as previously described [33]. Conditioned medium (CM) of hGluc-MSCs was harvested and filtered. CM $(5 \mu \mathrm{l})$ was then mixed with human serum (Atlanta Biologicals) with or without PBS dilution to final serum concentrations of $0 \%, 5 \%, 50 \%$, or $100 \%$, incubated at $37{ }^{\circ} \mathrm{C}$ at various times as indicated, and hGluc activity was measured with $20 \mu \mathrm{M} \mathrm{CTZ}$ (final concentration in a final volume of $200 \mu \mathrm{l})$. Mouse blood was collected as described [34] and added into $1 / 4$ volume of EDTA (Sigma-Aldrich) solution $(50 \mathrm{mM}, \mathrm{pH}=8.0)$. Blood $(5 \mu \mathrm{l})$ was mixed with $100 \mu \mathrm{l}$ of $100 \mu \mathrm{M} \mathrm{CTZ}$, and hGluc activity was measured immediately. All bioluminescent assays were performed with an IVIS Lumina (Caliper LifeSciences, Hopkinton, MA, USA) or a plate reader (BioTek, Winooski, VT, USA). All samples above were measured in triplicate.

\section{Cell implantation and imaging in vivo}

LV-Fluc-tdT MDA-MB-231 (Fluc-tdT-231) or LV-eGFP MDA-MB-231 (eGFP-231) breast cancer cells or LoVo colon cancer cells $\left(0.5 \times 10^{6} ; 2.5 \times 10^{6} / \mathrm{ml}\right.$ in DPBS) were implanted intravenously (i.v.) into nonobese diabetic/severe combined immunodeficiency gamma (NSG) mice (5 weeks, \#005557; The Jackson Laboratory, Bar Harbor, ME, USA). Five weeks later, in vivo Fluc activity from Fluc-tdT-231 cells was measured as described [35]. Briefly, in vivo Fluc signal was imaged with IVIS Lumina 10 minutes after intraperitoneal (i.p.) injection of D-luciferin $(150 \mathrm{mg} / \mathrm{kg}$ in DPBS; Lonza) into mice. hGluc-MSCs or Fluc-tdT-MSCs $\left(10^{6} ; 5 \times 10^{6} / \mathrm{ml}\right.$ in DPBS) were systemically infused into the mice harboring breast cancer cells and into healthy control mice. hGlucMSCs were labeled with the Dil lipophilic dye $\left(5 \mu \mathrm{l} / 10^{6}\right.$ cells; Life Technologies) by incubation at $37{ }^{\circ} \mathrm{C}$ for $20 \mathrm{mi}$ nutes before infusion. Mice were anesthetized with $2 \sim 3 \%$ of isoflurane (Western Medical Supply, Arcadia, CA, USA), and in vivo Fluc activity was measured at the indicated time points. Imaging was performed with the IVIS Lumina ( $\mathrm{n}=4$ in each case). All animal experiments and procedures were performed after the approval from the UCI Institution of Animal Care and Use Committee (protocol number 2012-3062) and conducted according to the Animal Welfare Assurance (\#A3416.01).

\section{Tissue processing and immunohistochemistry}

Tissues were collected and flash frozen in Tissue-Tek O.C.T ${ }^{\mathrm{ma}}$ Compound (Sakura Finetek, Torrance, CA, USA), with or without overnight fixation in $4 \%$ paraformaldehyde (Amresco, Solon, OH, USA), and with overnight incubation in $30 \%$ sucrose solution (Amresco). Sections $8 \mu \mathrm{m}$ thick were taken by cryostat and stained following an immunohistochemistry protocol for eGFP (sheep polyclonal IgG; Pierce Biotechnology, Rockford, IL, USA) and Fluc (rabbit polyclonal IgG; Abcam, 
Cambridge, UK). Briefly, slides were fixed in acetone (Thermo Fisher Scientific, Waltham, MA, USA) at $-20^{\circ}$ $\mathrm{C}$ for 10 minutes, permeabilized in $0.1 \%$ Triton X-100 (Sigma-Aldrich) for 10 minutes, and blocked in $0.1 \%$ Triton X-100 with $5 \%$ normal donkey serum (SigmaAldrich) for 30 minutes. Primary antibodies were diluted 1:100 from the stock solution in 0.05\% Tween-20 (Sigma-Aldrich) in PBS and applied overnight at $4{ }^{\circ} \mathrm{C}$. Slides were washed in $1 \mathrm{X}$ PBS, and then secondary antibodies (donkey anti-sheep IgG conjugated to Alexa Fluor 488, donkey anti-rabbit IgG conjugated to Alexa Fluor 594, Jackson ImmunoResearch Laboratories, West Grove, PA, USA) were diluted 1:500 from the stock solution in $0.05 \%$ Tween-20 in PBS and applied for 30 minutes at room temperature. TOTO-3 Iodide (2.4 $\mu \mathrm{M}$; Life Technologies) was added to the secondary antibody incubation. DAPI (4,6-diamidino-2-phenylindole) $(50 \mu \mathrm{g} / \mathrm{ml}$; Life Technologies) in PBS was applied to slides for 10 minutes before mounting. Slides were washed in PBS and mounted with DPX (Di-N-butyle phthalate in xylene) (Sigma-Aldrich) or Fluoromount-G (SouthernBiotech, Birmingham, AL, USA).

\section{Statistical analysis}

Data were analyzed by Student's $t$ test when comparing two groups and by analysis of variance when comparing more than two groups. Data were expressed as mean \pm standard deviation or as mean \pm standard error of the mean, and differences were considered significant at $P$ values of less than 0.05 .

\section{Results}

Humanized Gaussia luciferase is secreted from engineered MSCs in vitro and is stable and detectable in blood

Human bone marrow MSCs were stably transduced with lentivirus to express secreted humanized Gaussia luciferase (hGluc) as described above. To determine whether hGluc is secreted in an active form by MSC, cell-free CM was harvested from hGluc-MSCs 24 hours after MSC seeding at different concentrations (100, 1000, 2500 , or 5000 cells per $\mathrm{cm}^{2}$ ). The substrate CTZ was added and hGluc activity was measured for both cells and CM (Fig. 1a). hGluc activity increased with increasing cell number (Fig. 1a). In addition, hGluc activity in $\mathrm{CM}$ was 3- to 6-fold higher than inside cells (Fig. 1a),

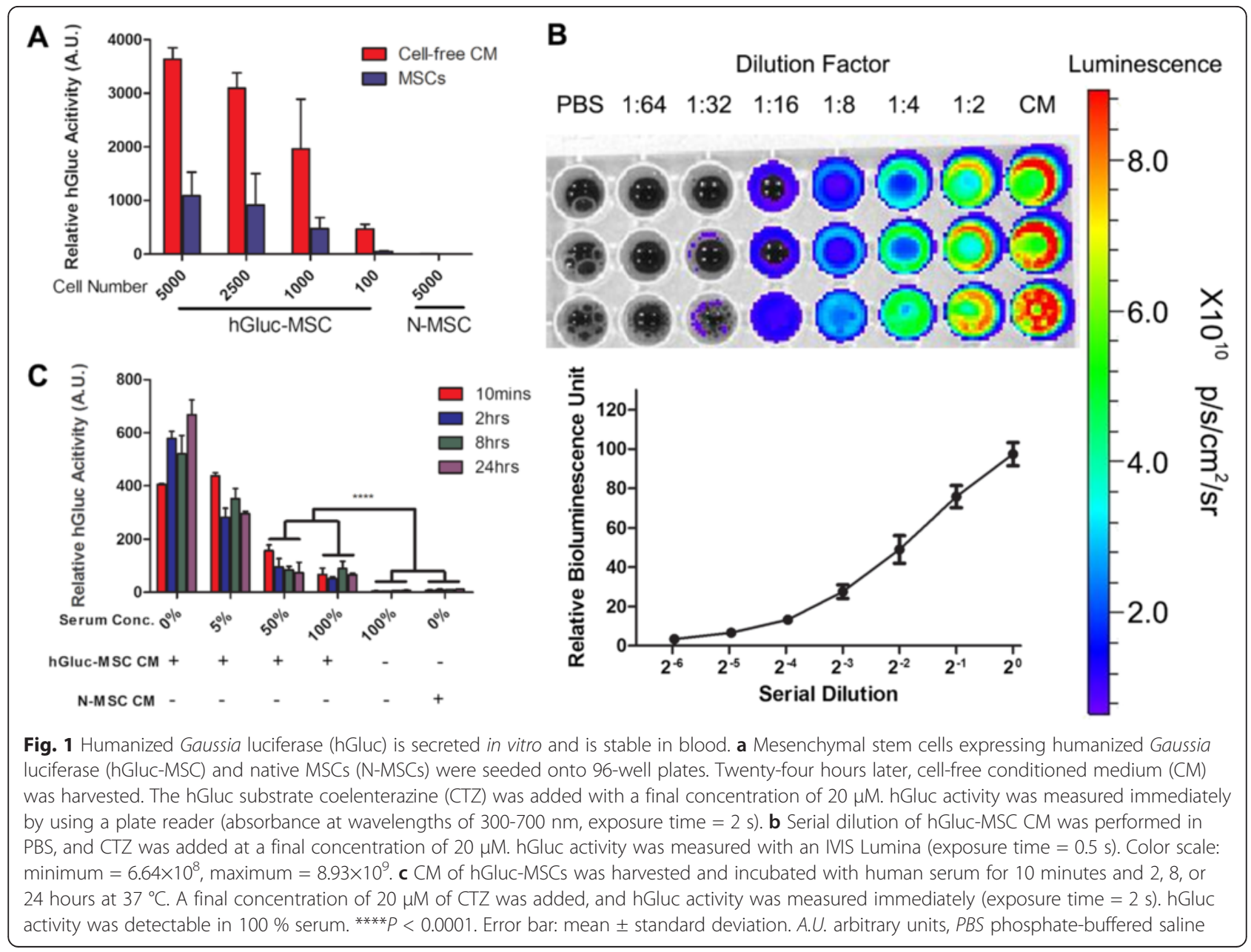




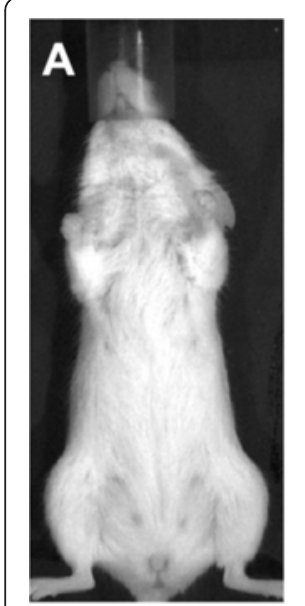

PBS

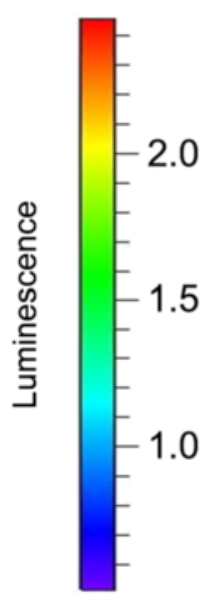

1.5

1.0

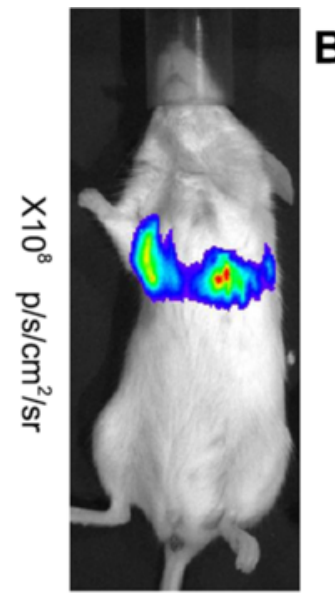

Fluc-tdT 231

B

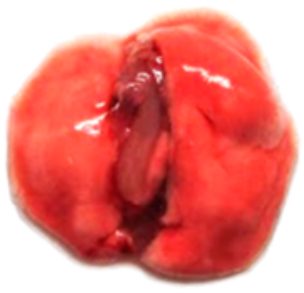

PBS

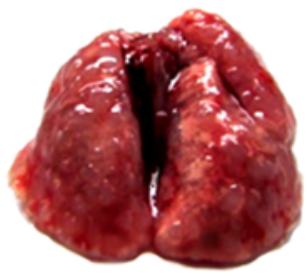

eGFP 231
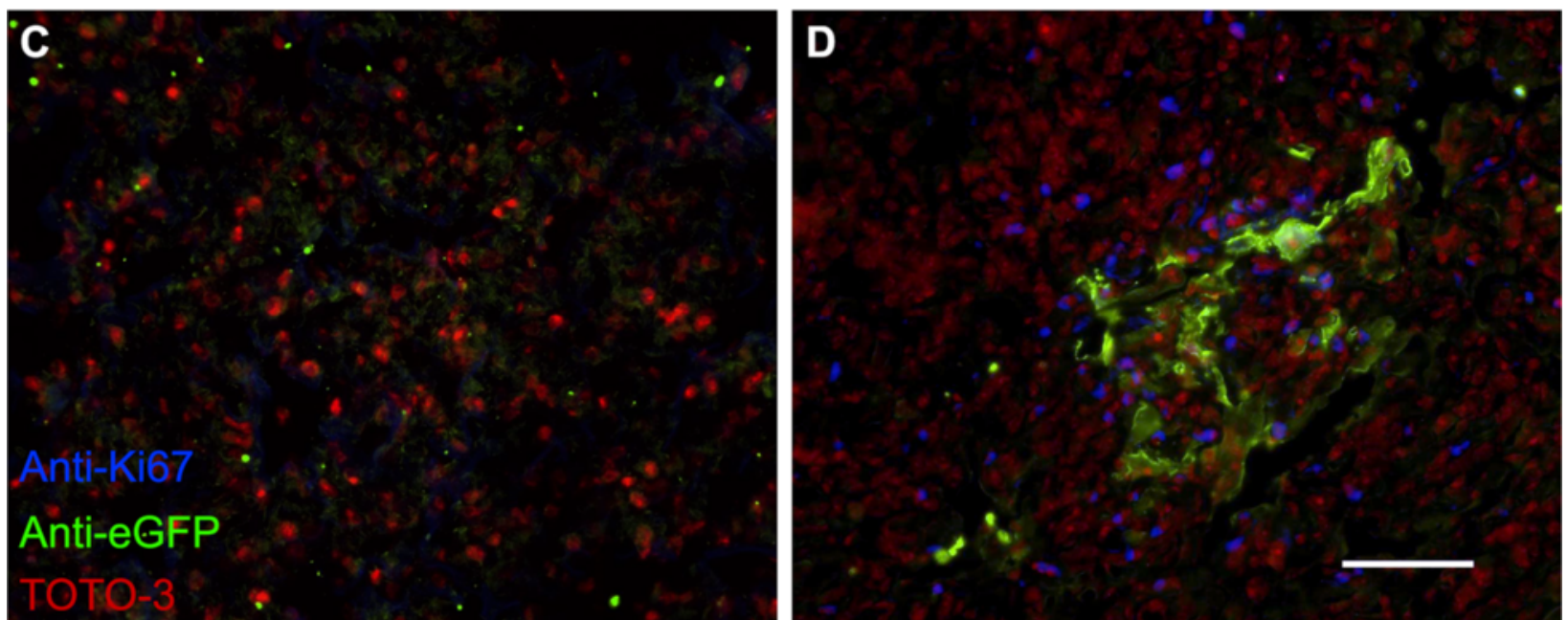

Fig. 2 Human-derived breast cancer was observed in xenotransplantation murine model. a Five weeks after $0.5 \times 10^{6}$ Fluc-tdT-231 were seeded i.v., NSG mice were injected intraperitoneally with D-Luciferin (150 mg/kg in Dulbecco's PBS) and in vivo Fluc activity was measured with IVIS Lumina 10 minutes after substrate administration. Exposure time $=5 \mathrm{~s}$. Color scale: minimum $=5.13 \times 10^{7}$, maximum $=2.46 \times 10^{8}$. $\mathbf{b}$ Representative pictures of tumor-free (left) and tumor-bearing (right) lungs. Eight weeks after MDA-MB-231 breast cancer cells or PBS were seeded i.v., NSG mice were euthanized and lungs were harvested. Frozen sections of lungs of c tumor-free mice and $\mathbf{d}$ eGFP-231 tumor-bearing mice sacrificed 5 weeks after cancer seeding were stained with anti-eGFP (green), anti-Ki67 (blue), and TOTO-3 (red). Scale bar: 50 Hm. eGFP enhanced green fluorescent protein, i.v. intravenously, NSG nonobese diabetic/severe combined immunodeficiency gamma, PBS phosphate-buffered saline

indicating that hGluc expressed by engineered MSCs is secreted in active form, as expected. hGluc-MSC CM was serially diluted with PBS and hGluc activity was measured in vitro and found to exhibit a linear function of concentration, in agreement with earlier reports [33, 36, 37] (Fig. 1b). To demonstrate whether luciferase activity from hGluc-MSCs is detectable and sufficiently stable in blood, human serum either directly (100 \%) or serially diluted in PBS was mixed with hGluc-MSCs CM. hGluc activity remained detectable $(P<0.0001)$ after 24 hours co-incubation and was not decreased significantly over time (Fig. 1c), indicating that hGluc-MSCs can be a stable marker in blood assays in vitro. Finally, since both firefly luciferase (Fluc-tdT) and
hGluc would be used in vivo (below), any potential cross-reactivity between Fluc-tdT and hGluc-MSCs was measured (Additional file 1: Figure S1). These two luciferases were substrate-specific and no cross-reaction was observed, as reported. Overall, these data show that hGluc expressed by engineered MSCs is secreted in vitro, is stable in human serum for up to 24 hours, and exhibits substrate-specific enzyme activity.

\section{Engineered MSCs home to tumor sites and persist longer} in the lungs of the tumor-bearing mice

As MSCs are reported to naturally home to tumor sites $[18,19]$, we tested this phenomenon in our experiment as a preliminary step to using MSCs that secrete hGluc 


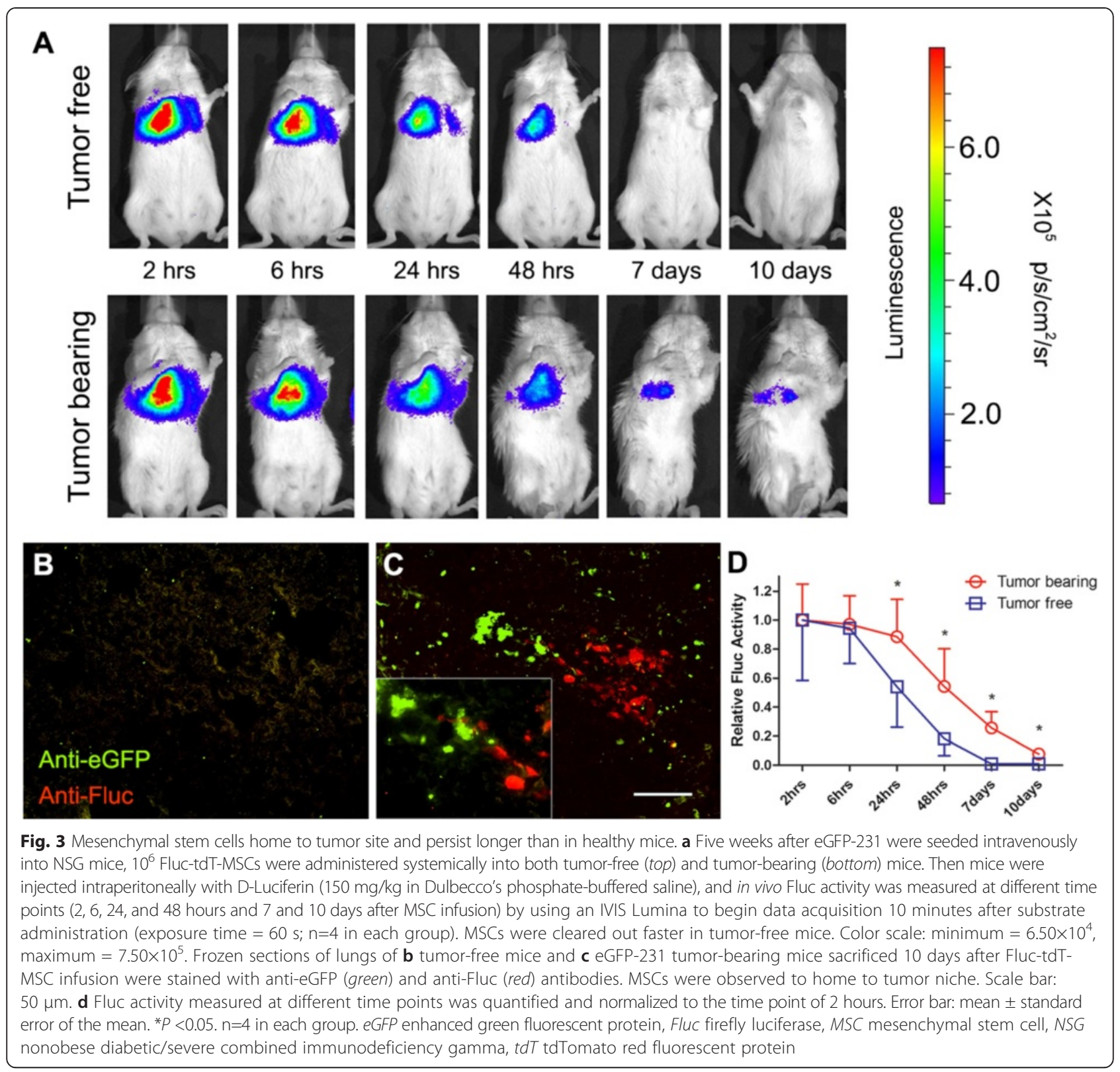

as a diagnostic tool for cancer detection and localization. Human breast cancer-derived MDA-MB 231 cells were labeled with eGFP or Fluc-tdT and implanted intravenously (i.v.) into immunodeficient NSG mice (Fig. 2) to establish a simple in vivo mouse model of breast cancer that has metastasized in the lungs [38, 39]. Tumor mass was observed in lungs both in vivo (Fig. $2 \mathrm{a}$ ) and ex vivo (Fig. 2b, d), whereas no tumor-related signal was seen in healthy lungs (Fig. 2a, c). Owing to the fact that hGluc is secreted by MSCs and to its diluted and limited signal under whole animal imaging conditions with IVIS Lumina [40] (data not shown), we used MSCs engineered with intracellular Fluc-tdT [41] for real-time imaging and localization of MSCs in tumors in situ. Fluc-tdT-MSCs were simultaneously labeled with red fluorescent protein (RFP) to assess Fluc transduction efficiency and to image any co-localized MSCs and tumor cells in subsequent ex vivo immunohistochemistry. Both Fluc activity and RFP signal from Fluc-tdT-MSCs were observed in vitro (Additional file 2: Figure S2), demonstrating that engineered MSCs express Fluc (Additional file 2: Figure S2A) with high transduction efficiency $\left(>90 \% \mathrm{RFP}^{+}\right.$; Additional file 2: Figure S2B-D).

To investigate any differences in MSCs homing between cancer-bearing and healthy mice, $10^{6}$ Fluc-tdT-MSCs were systemically infused into mice with or without breast cancer. Mice were anesthetized and in vivo Fluc activity was measured after i.p. administration of D-luciferin 


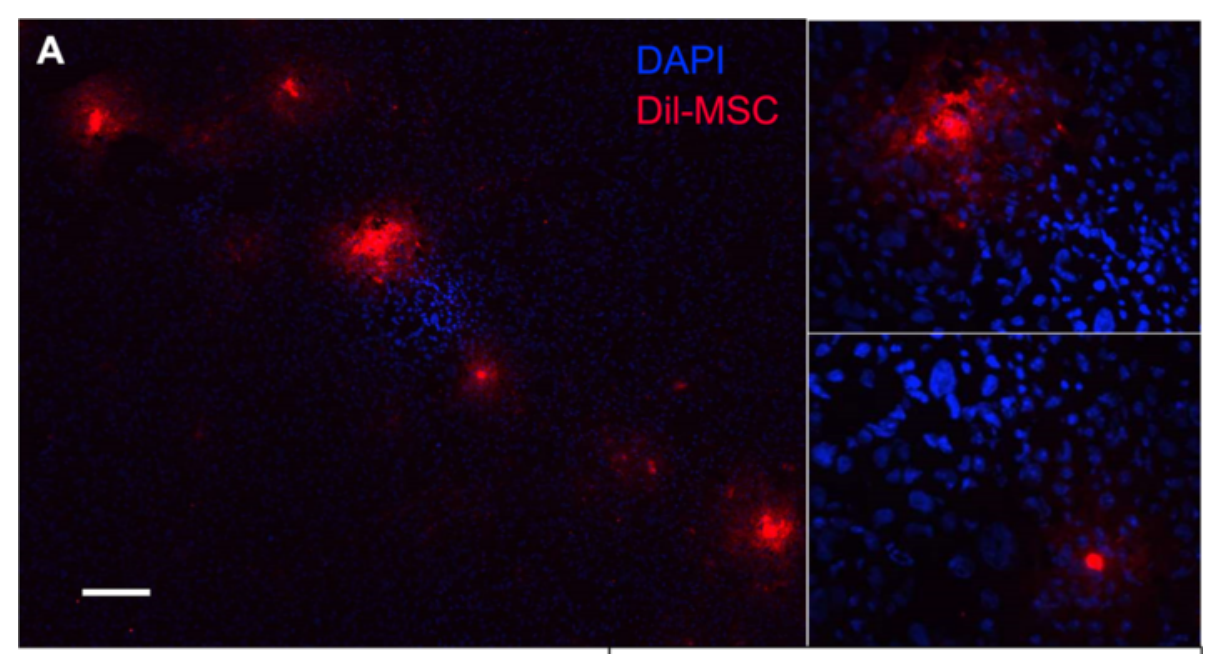

B
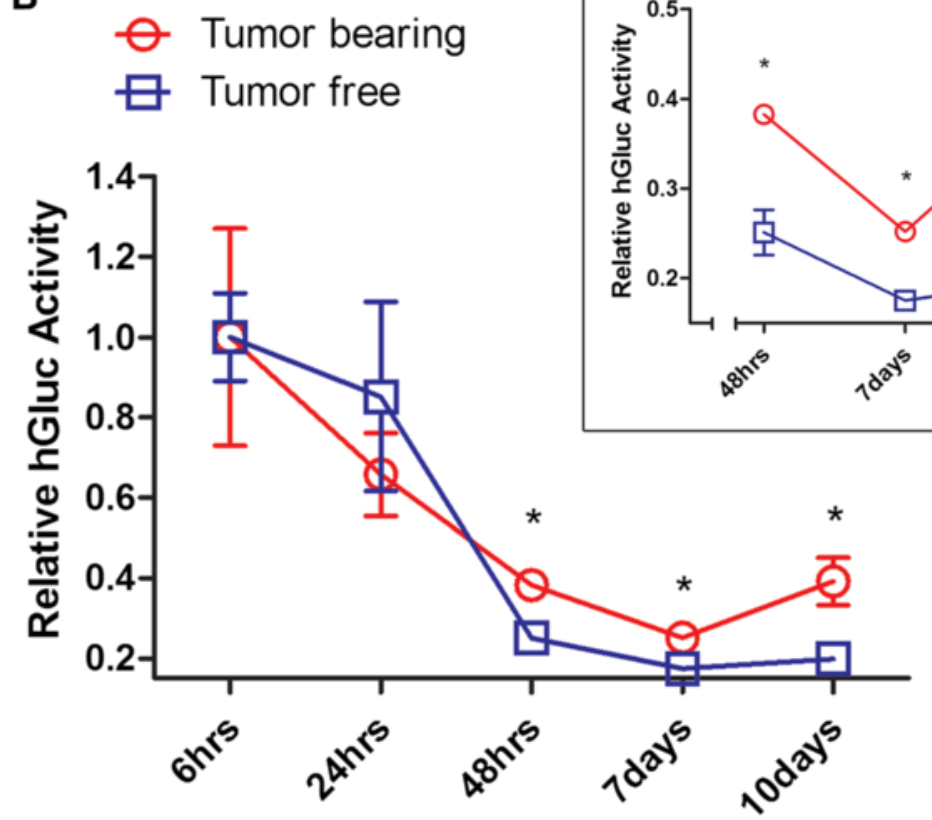

Fig. 4 Gaussia luciferase (hGluc) is active in murine blood and the signal is elevated in tumor-bearing mice. a Frozen sections of lungs of tumor-bearing mice sacrificed 10 days after Dil-labeled hGluc-MSC administration were stained with DAPI and then imaged by fluorescence microscopy. MSCs (red) were observed to home to tumor niche (dense blue). Scale bar: $100 \mu \mathrm{m}$. b Five weeks after Fluc-tdT-231 were seeded intravenously into NSG mice, $10^{6}$ hGluc-MSCs were administered systemically into both tumor-free and tumor-bearing mice. Then murine blood was harvested and hGluc activity was measured at different time points (6, 24, and 48 hours and 7 and 10 days after MSC infusion) with IVIS Lumina immediately after substrate was added. hGluc activity measured at different time points was quantified and normalized to the time point of 6 hours. The inset graph shows that the hGluc activity in blood between tumor-bearing and tumor-free mice is significantly different from 48 hours after MSC infusion. Error bar: mean \pm standard error of the mean. ${ }^{*} P<0.05$. Exposure time $=30 \mathrm{~s}$. $\mathrm{n}=4$ in each group. DAPI 4',6-Diamidino-2-phenylindole, MSC mesenchymal stem cell

substrate into mice at the indicated time points. In vivo imaging demonstrated that MSCs were detectable in tumor-bearing mice for as long as 10 days after systemic administration (Fig. 3a). Ex vivo immunohistochemistry data confirmed that engineered MSCs homed to the tumor niche in vivo (Figs. 3c and 4a). As we hypothesized, engineered MSCs persisted significantly longer in tumor-bearing lungs, especially at later time points (Fig. 3a). We then quantified the Fluc signal and found that significant differences between tumor-bearing and tumor-free mice emerged 24 hours after MSC infusion and lasted until 10 days after infusion (Fig. $3 \mathrm{~d}, \mathrm{n}=4, P<0.05$ ). To test whether our technology can be applied to other types of cancer, we investigated fused Fluc-tdT-MSCs into 
mice with lung metastasis of colon cancer. Similar results were observed (Additional file 3: Figure S3) which demonstrate engineered MSCs could home to and stay in tumor-bearing lungs for a significantly longer time compared with tumor-free lungs. Our data, along with mounting evidence of MSC tumor tropism in the literature $[18,22,42,43]$, suggest that the in vivo persistence of engineered MSCs in tumor-bearing compared with healthy animals provides a viable "marker" for broad cancer detection.

\section{hGluc secreted by engineered MSCs can be assayed in the blood of tumor-bearing mice}

We next investigated whether MSCs that were engineered to express hGluc can be used to detect metastasis of breast cancer to the lungs. hGluc was chosen as the reporter in this study because of its high sensitivity, lack of nonspecific cross-reactivity to other substrates (e.g., Additional file 1: Figure S1), and linear signal over a wide concentration range (Fig. 1b). In addition, hGluc has a short half-life in vivo (20 minutes), allowing repeated real-time testing without undesirable excessive signal accumulation, but a long half-life in vitro (6 days), allowing convenient sample storage [33]. As hGluc is secreted, it cannot be used as a marker to co-localize MSCs and tumor as seen in Fig. 3c for intracellular Fluc. Therefore, in this set of experiments, we stained hGlucMSCs with the Dil lipophilic dye before they were infused i.v. into mice. Like Fluc-tdT-MSCs, Dil-MSCs were detectable in the tumor niche up to 10 days postinfusion (Fig. 4a). Mouse blood was collected at the indicated time points, and hGluc activity was measured. Although the detected signal decayed rapidly over time as expected, the difference of hGluc activity in blood between tumor-bearing and tumor-free mice was significant starting from 48 hours after MSC administration and lasting until 10 days post-infusion (Fig. 4b), suggesting that systemically infused hGluc-MSC can be used for the potential development of a simple blood assay for cancer detection in this murine model. In summary, this set of data supports the feasibility of using engineered MSCs with secreted hGluc as a blood test for the presence of cancer.

\section{Discussion}

Early detection of cancer, especially metastasis, is a necessary and often critical first step to effectively treat and eradiate cancer. Traditional imaging tools and molecular biomarker-based assays are typically complex, expensive, and/or invasive for routine screening for most cancers; most importantly, they frequently do not possess the sensitivity and specificity to identify heterogeneous cancers at early stages. In our study, we developed a stem cell-based detection system that can detect cancer, including metastases, by collecting small amounts of blood with a minimally invasive procedure. Our engineered MSCs could home to tumor sites and persist there for significantly longer durations compared with healthy mice. The signal derived from engineered stem cells lasted longer compared with current imaging tracers [5], and no repeat administration was needed. With one single administration, the presence of tumor could be monitored continuously through a prolonged period of time, making MSCs a convenient tool for real-time cancer detection. Compared with acellular systems (e.g., antibodies and nanoparticles), the natural interactions between MSCs and tumor involve complex adaptive sensing and responding systems that enable more efficient and specific reporting of cancer and metastases. This intrinsic biological property of tumor homing therefore potentially allows our stem cell approach to "universally" identify many cancers regardless of their origins, types, and anatomical sites. In addition, stem cell-based probe delivery circumvents many hurdles associated with passive delivery (i.e., by direct administration or polymeric nanoparticles via the EPR effect), including penetrating the endothelium and the increased pressure associated with tumors. In addition, the use of distinct, exogenous markers (hGluc in this article) as surrogate markers to detect and monitor cancer is more advantageous than endogenous markers because of the lack of unique cancer biomarkers. In our assay, a positive detection of hGluc (even with a small signal) would indicate the presence of cancer, which therefore helps to eliminate the need for sophisticated signal normalization over background as required in conventional cancer detection assays. Therefore, our simple, noninvasive stem cell-based blood test might be useful for routine cancer screening, detecting small tumors and metastases, and monitoring cancer progression and recurrence during the course of treatment.

Since MSCs possess not only tumor tropism but also tropism for bone marrow and sites of inflammation and injury $[20,23]$, it remains important to distinguish those conditions from cancer when using MSC-based methods to detect cancer. In addition, given high cancer heterogeneity, our next-generation systems aim to engineer MSCs with activatable, cancer type-specific probes to further increase the assay specificity. The long-term goal is to establish a panel of tests that can effectively discriminate between cancer (sub)types and stages and distinguish between cancer and other disorders that share similar symptoms, including inflammation and injury.

MSCs were chosen in our current (first-generation) system because they can be easily obtained from multiple adult tissues [44], including bone marrow and fat, therefore avoiding ethical concerns. MSCs are also relatively easy to expand in culture and can be readily engineered to express functional therapeutics or reporters [14, 23]. Importantly, the clinically approved Prochymal and hundreds of other 
ongoing clinical trials have demonstrated that allogeneic MSCs are generally safe for use in the human without harsh immunosuppressive regimens. Nonetheless, as MSCs themselves may participate in cancer progression or regression [22], further considerations are required. The interactions between MSCs and cancer remain incompletely understood [14, 22], with different reports indicating conflicting findings from endogenous and exogenous MSCs on cancer progression $[22,45,46]$. Thus, safety tests and optimizations will likely be required to better control the fate of our engineered MSCs after cancer detection, though no obvious MSC-mediated cancer growth was observed within our detection window (Additional file 4: Figure S4). To mitigate this potential issue, for example, a suicide gene [47] can be engineered into our MSC-based system so that after completion of the cancer detection test, the remaining engineered MSCs can be eliminated by using exogenously administered drugs. For example, inducible human caspase-9 (iC9), which can be activated by a bio-inert small-molecule drug, has been used as a safety switch in clinical trials of cell therapy with limited immunogenicity [48]. Another limitation of our study is that we used a relatively large tumor burden as our model to demonstrate our proof-of-concept because of its technical simplicity. In the future, we will evaluate our engineered stem cell approach to detect early-stage cancer and metastases when they are small by using cancer models with smaller tumor burden by either reducing the cell number administered or at the early stages of the beast cancer progression. These future experiments will allow us to determine the smallest tumor size we can detect with our technology. Furthermore, our system may be used as companion diagnostics combined with other treatments, for example, identifying certain patients and monitoring side effects. Finally, our cell-based blood assay may represent a new platform for monitoring the fate and functions of transplanted cells as well as for assessing the in vivo microenvironment where they reside.

\section{Conclusions}

We demonstrate for the first time, to the best of our knowledge, a simple blood test for cancer detection. This test is based on the premise of exploiting the natural tumor-homing ability of MSCs to further engineer them to express a secreted luciferase with optimal biocompatibility and kinetic parameters. Similar to our current murine studies, these "reporter MSCs" could be developed to identify the presence of small tumors or metastases in humans that would otherwise be undetectable by existing imaging modalities. We hope this simple "off the shelf" allogeneic stem cell-based diagnostic test can be used to screen, detect, and monitor cancer on a routine basis.

\section{Note}

This article is part of an 'Emerging Investigators' collection

showcasing the work of early career investigators who have

demonstrated growing leadership in the field of stem cells and

regenerative medicine. Other articles in the series can be found

online at http://stemcellres.com/series/emerginginvestigators.

\section{Additional files}

Additional file 1: Figure S1. Firefly and humanized Gaussia luciferases are substrate-specific and not cross-reactive. hGluc-MSCs, Fluc-tdT-MSCs (Fluc-MSCs), and N-MSCs were seeded in 96-well plate. The firefly luciferase substrate D-luciferin (final concentration $=150 \mu \mathrm{g} / \mathrm{ml}$ ) or the humanized Gaussia luciferase substrate CTZ (final concentration $=20 \mu \mathrm{M}$ ) was added, and luciferase activity was measured with a plate reader. Error bar: mean \pm standard deviation. Exposure time $=2 \mathrm{~s}$. A.U. arbitrary units, CTZ coelenterazine, Fluc firefly luciferase, hGluc humanized Gaussia luciferase, MSC mesenchymal stem cell, tdT tdTomato red fluorescent protein. (PNG $37 \mathrm{~kb}$ )

Additional file 2: Figure S2. Engineered mesenchymal stem cells (Fluc-tdT-MSCs) express firefly luciferase (Fluc) and red fluorescent protein (tdT). (A) Fluc-tdT-MSCs (Fluc-MSCs) were seeded onto 96-well plate, and 24 hours later D-luciferin was added at a final concentration of $150 \mathrm{\mu g} / \mathrm{ml}$. Fluc activity was measured with a plate reader. Error bar: mean \pm standard deviation. Exposure time $=2 \mathrm{~s}$. ${ }^{* * *} P<0.001$. (B-D) Fluc-tdT-MSC were imaged by fluorescence microcopy 24 hours after seeding. Scale bar: $50 \mu$ m. A.U. arbitrary units, MSC mesenchymal stem cell, $t d T$ tdTomato red fluorescent protein. (PNG $6051 \mathrm{~kb}$ )

Additional file 3: Figure S3. Systemically infused MSCs persist in the lungs of the LoVo cancer cell-bearing mice. Five weeks after LoVo colon cancer cells were seeded intravenously into NSG mice, $10^{6}$ Fluc-tdT-MSCs were administered systemically into both tumor-free (blue) and tumor-bearing (red) mice. Then mice were injected intraperitoneally with D-Luciferin (150 mg/kg in Dulbecco's phosphate-buffered saline), and in vivo Fluc activity was measured at different time points $(6,24$, and 48 hours and 7 and 10 days after MSC infusion) by using an IVIS Lumina to begin data acquisition 10 minutes after substrate administration (exposure time $=60 \mathrm{~s}$ ). Fluc activity measured at different time points was quantified. Similar to the results with MDA-MB-231 breast cancer cells, MSCs were cleared out faster in tumor-free mice, showing that the tumor tropism of MSCs is applicable to multiple types of cancers. Error bar: mean \pm standard error of the mean. ${ }^{*} P<0.05$. $n=4$ for tumor-bearing mice and $n=3$ for tumor-free mice. Fluc firefly luciferase, MSC mesenchymal stem cell, NSG nonobese diabetic/severe combined immunodeficiency gamma, $t d T$ tdTomato red fluorescent protein. (PNG $128 \mathrm{~kb}$ )

Additional file 4: Figure S4. Engineered mesenchymal stem cell (hGluc-MSC) infusion has no influence on the growth of cancer metastasis size in vivo. Five weeks after Fluc-tdT-231 were seeded intravenously into NSG mice, $10^{6}$ hGluc-MSCs or PBS was administered systemically into tumor-bearing mice (day 0). In vivo Fluc activity was measured with IVIS Lumina 10 minutes after substrate administration before (day 0 ) and 10 days after MSC infusion (day 10). Exposure time $=5 \mathrm{~s}$. Relative metastasis index (RMI) $=$ Luciferase activity on day 10 (after) / Luciferase activity on day 0 (before). $N=4$ for each group. $h$ Gluc humanized Gaussia luciferase, MSC mesenchymal stem cell, n.s. not significant, NSG nonobese diabetic/severe combined immunodeficiency gamma, PBS phosphate-buffered saline, $t d T$ tdTomato red fluorescent protein. (PNG $126 \mathrm{~kb})$

\section{Abbreviations}

ATCC: American Type Culture Collection; BRCA1/2: Breast cancer 1/2; CM: Conditioned medium; CT: Computed tomography; CTZ: Coelenterazine; CXCL12: C-X-C motif chemokine 12; DPBS: Dulbecco's phosphate-buffered 
saline; EDTA: Ethylenediaminetetraacetic acid; eGFP: Enhanced green fluorescent protein; EPR: Enhanced permeability and retention; FBS: Fetal bovine serum; Fluc: Firefly luciferase; hGluc: Humanized Gaussia luciferase; i.p.: Intraperitoneal; i.v.: Intravenously; LV: Lentiviral; MRI: Magnetic resonance imaging; MSC: Mesenchymal stem cell; NSG: Nonobese diabetic/severe combined immunodeficiency gamma; PBS: Phosphate-buffered saline; PenStrep: penicillin-streptomycin; PET: Positron emission tomography; RFP: Red fluorescent protein; tdT: tdTomato red fluorescent protein; UCI: University of California, Irvine.

\section{Competing interests}

The authors declare that they have no competing interests.

\section{Authors' contributions}

LL designed the study, carried out the in vitro and in vivo experiments, processed tissues, analyzed data, interpreted the results, and drafted the manuscript. SXZ processed tissues, analyzed data, prepared figures, and drafted the manuscript. RA carried out the in vitro bioluminescence assay, analyzed data, and participated in manuscript drafting. WL carried out the in vivo experiments, analyzed the data, and participated in manuscript editing. ML carried out the cell engineering and participated in tissue processing, figure preparation, and drafting the manuscript. GP participated in the in vivo imaging, processed tissues, analyzed the data, and revised the manuscript. EJP participated in designing the study and interpreting the results and edited the manuscript. WZ was responsible for conception and design of the research, interpreted the results, and revised the manuscript. All authors read and approved the manuscript.

\section{Authors' information}

$\mathrm{LL}, \mathrm{SXZ}, \mathrm{RA}$, and $\mathrm{ML}$ are graduate student researchers in pharmaceutical sciences, biomedical engineering, and biotechnology at UCI. EJP and WL are project scientists in stem cells and immunology at UCI. GP is a student intern at the California Institute for Regenerative Medicine. WZ is the principal investigator of the project at $\mathrm{UCl}$.

\section{Acknowledgments}

This work was supported by the National Institutes of Health (1DP2CA195763-01) and in part by the American Heart Association (13BGIA17140099). SXZ was supported by a Cardiovascular Applied Research and Entrepreneurship fellowship (NIH/NHLBI T32). WL was supported by a California Institute for Regenerative Medicine (CIRM) fellowship (TG2-01152), and GP was supported by a CIRM training grant (TB1-01182).

\section{Author details}

'Department of Pharmaceutical Sciences, University of California, Irvine, 845 Health Sciences Road, Irvine, CA 92697, USA. ${ }^{2}$ Department of Biomedical Engineering, University of California, Irvine, 845 Health Sciences Road, Irvine, CA 92697, USA. ${ }^{3}$ Sue and Bill Gross Stem Cell Research Center, University of California, Irvine, 845 Health Sciences Road, Irvine, CA 92697, USA. ${ }^{4}$ Chao Family Comprehensive Cancer Center, University of California, Irvine, 845 Health Sciences Road, Irvine, CA 92697, USA. ${ }^{5}$ Edwards Lifesciences Center for Advanced Cardiovascular Technology, University of California, Irvine, 845 Health Sciences Road, Irvine, CA 92697, USA. ${ }^{6}$ Department of Biological Sciences, California State University, Long Beach, 1250 Bellflower Boulevard, Long Beach, CA 90840, USA.

\section{Received: 21 January 2015 Revised: 5 February 2015} Accepted: 11 August 2015 Published online: 21 September 2015

\section{References}

1. Hanahan D, Weinberg RA. Hallmarks of cancer: the next generation. Cell. 2011;144:646-74. doi:10.1016/j.cell.2011.02.013.

2. Hussain T, Nguyen QT. Molecular imaging for cancer diagnosis and surgery. Adv Drug Deliv Rev. 2014;66:90-100. doi:10.1016/j.addr.2013.09.007.

3. Hedley BD, Chambers AF. Tumor dormancy and metastasis. Adv Cancer Res. 2009;102:67-101. doi:10.1016/S0065-230X(09)02003-X.

4. Mehlen P, Puisieux A. Metastasis: a question of life or death. Nat Rev Cancer. 2006;6:449-58. doi:10.1038/nrc1886.

5. Kapoor V, McCook BM, Torok FS. An introduction to PET-CT imaging. Radiographics. 2004;24:523-43. doi:10.1148/rg.242025724.
6. Schick F. Whole-body MRI, at high field: technical limits and clinical potential. Eur Radiol. 2005;15:946-59. doi:10.1007/s00330-005-2678-0

7. Buchberger W, Niehoff A, Obrist P, DeKoekkoek-Doll P, Dunser M. Clinically and mammographically occult breast lesions: detection and classification with high-resolution sonography. Semin Ultrasound CT MR. 2000;21:325-36.

8. Warren AD, Gaylord ST, Ngan KC, Dumont Milutinovic M, Kwong GA, Bhatia $\mathrm{SN}$, et al. Disease detection by ultrasensitive quantification of microdosed synthetic urinary biomarkers. J Am Chem Soc. 2014;136:13709-14. doi:10.1021/ja505676h.

9. Kwong GA, von Maltzahn G, Murugappan G, Abudayyeh O, Mo S, Papayannopoulos IA, et al. Mass-encoded synthetic biomarkers for multiplexed urinary monitoring of disease. Nat Biotechnol. 2013;31:63-70. doi:10.1038/nbt.2464.

10. Danino T, Prindle A, Kwong GA, Skalak M, Li H, Allen K, et al. Programmable probiotics for detection of cancer in urine. Sci Transl Med. 2015;7:289ra84. doi:10.1126/scitranslmed.aaa3519.

11. Hanash SM, Baik CS, Kallioniemi O. Emerging molecular biomarkers-bloodbased strategies to detect and monitor cancer. Nat Rev Clin Oncol. 2011;8:142-50. doi:10.1038/nrclinonc.2010.220.

12. Schroder FH, Hugosson J, Roobol MJ, Tammela TL, Ciatto S, Nelen V, et al. Prostate-cancer mortality at 11 years of follow-up. N Engl J Med. 2012:366:981-90. doi:10.1056/NEJMoa1113135.

13. Tomasetti C, Vogelstein B. Cancer etiology. Variation in cancer risk among tissues can be explained by the number of stem cell divisions. Science. 2015;347:78-81. doi:10.1126/science.1260825.

14. Ankrum J, Karp JM. Mesenchymal stem cell therapy: two steps forward, one step back. Trends Mol Med. 2010;16:203-9. doi:10.1016/j.molmed.2010.02.005.

15. Shah K. Mesenchymal stem cells engineered for cancer therapy. Adv Drug Deliv Rev. 2012;64:739-48. doi:10.1016/j.addr.2011.06.010.

16. Loebinger MR, Janes SM. Stem cells as vectors for antitumour therapy. Thorax. 2010;65:362-9. doi:10.1136/thx.2009.128025.

17. Studeny M, Marini FC, Dembinski JL, Zompetta C, Cabreira-Hansen M, Bekele BN, et al. Mesenchymal stem cells: potential precursors for tumor stroma and targeted-delivery vehicles for anticancer agents. J Natl Cancer Inst. 2004;96:1593-603. doi:10.1093/jnci/djh299.

18. Kidd S, Spaeth E, Dembinski JL, Dietrich M, Watson K, Klopp A, et al. Direct evidence of mesenchymal stem cell tropism for tumor and wounding microenvironments using in vivo bioluminescent imaging. Stem Cells. 2009;27:2614-23. doi:10.1002/stem.187.

19. Reagan MR, Kaplan DL. Concise review: Mesenchymal stem cell tumor-homing: detection methods in disease model systems. Stem Cells. 2011;29:920-7. doi:10.1002/stem.645.

20. Zhao W, Phinney DG, Bonnet D, Dominici M, Krampera M. Mesenchymal stem cell biodistribution, migration, and homing in vivo. Stem Cells Int. 2014;2014:292109. doi:10.1155/2014/292109.

21. Ankrum JA, Ong JF, Karp JM. Mesenchymal stem cells: immune evasive, not immune privileged. Nat Biotechnol. 2014;32:252-60. doi:10.1038/nbt.2816.

22. Droujinine IA, Eckert MA, Zhao W. To grab the stroma by the horns: from biology to cancer therapy with mesenchymal stem cells. Oncotarget. 2013;4:651-64

23. Karp JM, Leng Teo GS. Mesenchymal stem cell homing: the devil is in the details. Cell Stem Cell. 2009;4:206-16. doi:10.1016/j.stem.2009.02.001.

24. Bexell D, Scheding S, Bengzon J. Toward brain tumor gene therapy using multipotent mesenchymal stromal cell vectors. Mol Ther. 2010;18:1067-75. doi:10.1038/mt.2010.58

25. Phinney DG, Prockop DJ. Concise review: Mesenchymal stem/multipotent stromal cells: The state of transdifferentiation and modes of tissue repair Current views. Stem Cells. 2007;25:2896-902. doi:10.1634/stemcells.2007-0637.

26. Liu L, Eckert MA, Riazifar H, Kang DK, Agalliu D, Zhao W. From blood to the brain: can systemically transplanted mesenchymal stem cells cross the blood-brain barrier? Stem Cells Int. 2013;2013:435093. doi:10.1155/2013/435093.

27. Hingtgen SD, Kasmieh R, van de Water J, Weissleder R, Shah K. A novel molecule integrating therapeutic and diagnostic activities reveals multiple aspects of stem cell-based therapy. Stem Cells. 2010;28:832-41. doi:10.1002/stem.313.

28. Chung E, Yamashita H, Au P, Tannous BA, Fukumura D, Jain RK. Secreted Gaussia luciferase as a biomarker for monitoring tumor progression and treatment response of systemic metastases. PLoS One. 2009;4, e8316. doi:10.1371/journal.pone.0008316.

29. Tannous BA, Teng J. Secreted blood reporters: insights and applications. Biotechnol Adv. 2011;29:997-1003. doi:10.1016/j.biotechadv.2011.08.021. 
30. Bovenberg MS, Degeling MH, Tannous BA. Enhanced Gaussia luciferase blood assay for monitoring of in vivo biological processes. Anal Chem. 2012;84:1189-92. doi:10.1021/ac202833r.

31. El-Amouri SS, Cao P, Miao C, Pan D. Secreted luciferase for in vivo evaluation of systemic protein delivery in mice. Mol Biotechnol. 2013;53:63-73. doi:10.1007/s12033-012-9519-6.

32. Dull T, Zufferey R, Kelly M, Mandel RJ, Nguyen M, Trono D, et al. A third-generation lentivirus vector with a conditional packaging system. J Virol. 1998;72:8463-71.

33. Tannous BA. Gaussia luciferase reporter assay for monitoring biological processes in culture and in vivo. Nat Protoc. 2009;4:582-91. doi:10.1038/nprot.2009.28.

34. Donovan J, Brown P. Blood collection. Curr Protoc Neurosci. 2005;Appendix 4:Appendix 4G. doi:10.1002/0471142301.nsa04gs33.

35. Kong Y, Shi Y, Chang M, Akin AR, Francis KP, Zhang N, et al. Whole-body imaging of infection using bioluminescence. Curr Protoc Microbiol. 2011;Chapter 2:Unit 2C 4. doi:10.1002/9780471729259.mc02c04s21.

36. Tannous BA, Kim DE, Fernandez JL, Weissleder R, Breakefield XO. Codonoptimized Gaussia luciferase cDNA for mammalian gene expression in culture and in vivo. Mol Ther. 2005;11:435-43. doi:10.1016/j.ymthe.2004.10.016.

37. Welsh JP, Patel KG, Manthiram K, Swartz JR. Multiply mutated Gaussia luciferases provide prolonged and intense bioluminescence. Biochem Biophys Res Commun. 2009;389:563-8. doi:10.1016/j.bbrc.2009.09.006.

38. Fantozzi A, Christofori G. Mouse models of breast cancer metastasis. Breast Cancer Res. 2006;8:212. doi:10.1186/bcr1530.

39. Wang CY, Brown PH. Animal Models for Breast Cancer Prevention Research. Genetically Engineered Mice for Cancer Research: Design, Analysis, Pathways, Validation and Pre-Clinical Testing. 2012:497-526. doi:10.1007/978-0-387-69805-2_24.

40. Close DM, Xu T, Sayler GS, Ripp S. In vivo bioluminescent imaging (BLI): noninvasive visualization and interrogation of biological processes in living animals. Sensors (Basel). 2011;11:180-206. doi:10.3390/s110100180.

41. Prescher JA, Contag CH. Guided by the light: visualizing biomolecular processes in living animals with bioluminescence. Curr. Opin. Chem. Biol. 2010;14:80-89. doi:10.1016/j.cbpa.2009.11.001.

42. Elman JS, Murray RC, Wang F, Shen K, Gao S, Conway KE, et al. Pharmacokinetics of natural and engineered secreted factors delivered by mesenchymal stromal cells. PLoS One. 2014;9, e89882. doi:10.1371/journal.pone.0089882.

43. Shah K. Stem cell therapeutics for cancer. Hoboken, NJ: John Wiley \& Sons; 2013.

44. Caplan Al, Correa D. The MSC: an injury drugstore. Cell Stem Cell. 2011;9:11-5. doi:10.1016/j.stem.2011.06.008

45. El-Haibi CP, Bell GW, Zhang J, Collmann AY, Wood D, Scherber CM, et al. Critical role for lysyl oxidase in mesenchymal stem cell-driven breast cancer malignancy. Proc Natl Acad Sci U S A. 2012;109:17460-5. doi:10.1073/pnas.1206653109.

46. Li HJ, Reinhardt F, Herschman HR, Weinberg RA. Cancer-stimulated mesenchymal stem cells create a carcinoma stem cell niche via prostaglandin E2 signaling. Cancer Discov. 2012;2:840-55. doi:10.1158/2159-8290.CD-12-0101.

47. Aboody KS, Najbauer J, Metz MZ, D'Apuzzo M, Gutova M, Annala AJ, et al. Neural stem cell-mediated enzyme/prodrug therapy for glioma: preclinical studies. Sci Transl Med. 2013;5:184ra59. doi:10.1126/scitransImed.3005365.

48. Zhou X, Dotti G, Krance RA, Martinez CA, Naik S, Kamble RT, et al. Inducible caspase-9 suicide gene controls adverse effects from alloreplete T cells after haploidentical stem cell transplantation. Blood. 2015;125:4103-13. doi:10.1182/blood-2015-02-628354.

\section{Submit your next manuscript to BioMed Central and take full advantage of:}

- Convenient online submission

- Thorough peer review

- No space constraints or color figure charges

- Immediate publication on acceptance

- Inclusion in PubMed, CAS, Scopus and Google Scholar

- Research which is freely available for redistribution 